\title{
Absolute Continuity of Hamiltonian Operators with Repulsive Potentials
}

\author{
By \\ Masaharu ArAi*
}

\section{Introduction}

The purpose of the present note is to improve the results of R.B. Lavine [3] on the absolute continuity of a Hamiltonian operator $H=-\Delta$ $+V$ in $L_{2}\left(R^{n}\right)$ with repulsive potential $V$ (where $\Delta$ is the Laplacian and $V$ is the operation of multiplication by a real function $V(x))$. If the potential $V(x)$ satisfies

$$
\partial V / \partial r \leqq 0
$$

where $r=|x|$, then it is said to be repulsive.

Lavine [3] shows that if the potential $V$ satisfies not only the assumption (1) but also

$$
\partial V / \partial r \leqq-a r^{-3+\varepsilon} \quad \text { for large } r
$$

for some positive constants $a$ and $\varepsilon$, then $H=-\Delta+V$ is absolutely continuous for $n=1,3$. Our aim is to extend his results in two directions: One is to remove the restriction on the dimension $n$ of the space, and the other is to remove the assumption (2). This will be accomplished except for the cases $n=1$ and 2, where we must impose an assumption somewhat weaker than (2).

Our method is that of Lavine [3] which is based on an abstract theory of Putnam [4] on commutators of pairs of selfadjoint operators.

Received July 15, 1971.

Communicated by S. Matsuura.

* Faculty of Economics, Ritsumeikan University, Tōjiin, Kyoto, 603, Japan. 


\section{Notations and Results}

Let $T$ be a selfadjoint operator in a Hilbert space $\mathfrak{S}$ and $E(\lambda)$ be the spectral family associated with $T$. Denote by $\mathfrak{S}_{a c}(T)$ the set of all vectors $\phi$ such that $\|E(\lambda) \phi\|^{2}$ is absolutely continuous with respect to the Lebesgue measure. Then $\mathfrak{S}_{a c}(T)$ is a closed subspace which reduces $T$; cf. [2], Chapter X, Theorem 1.5. Denote by $T_{a c}$ the restriction of $T$ in $\mathfrak{S}_{a c}(T)$. The spectrum of $T_{a c}$ is called the absolutely continuous spectrum of $T$. If $T=T_{a c}$, that is, $\mathfrak{S}_{a c}(T)=\mathfrak{E}$, then we say that $T$ is absolutely continuous.

Let $\alpha=\left(\alpha_{1}, \alpha_{2}, \ldots, \alpha_{n}\right)$ be a vector with nonnegative integral coordinates and $|\alpha|=\alpha_{1}+\alpha_{2}+\cdots+\alpha_{n}$. We denote by $D^{\alpha} \phi$ the partial derivative

$$
D^{\alpha} \phi=\frac{\partial^{|\alpha|} \phi}{\partial^{\alpha_{1}} x_{1} \partial^{\alpha_{2}} x_{2} \cdots \partial^{\alpha_{n}} x_{n}}
$$

in the distribution sense.

Let $X$ be a set of functions defined in a domain $\Omega \subset R^{n}$. We denote by $\varepsilon_{X}^{p}(\Omega)$ the set of all functions $\phi$ such that all the derivatives $D^{\alpha} \phi$ in the distribution sence for $0 \leqq|\alpha| \leqq p$ belong to the set $X^{1}{ }^{1}$ ) In case $\Omega=$ $R^{n}$, we sometimes write $\varepsilon_{X}^{p}$ instead of $\varepsilon_{X}^{p}\left(R^{n}\right)$.

Let $\mathfrak{C}=L_{2}\left(R^{n}\right)$ be the Hilbert space with the ordinary inner product

$$
(\phi, \psi)=\int \phi(x) \psi(x)^{*} d x
$$

where the asterisk means the complex conjugate. Let $H_{0}$ be the selfadjoint operator $H_{0}=-\Delta$ with domain $D\left(H_{0}\right)=\varepsilon_{L_{2}}^{2}$.

Let $Q_{\alpha}(\alpha>0)$ be the set of real functions $V(x)$ satisfying the assumption

$$
\begin{aligned}
& \int_{|x-y| \leqq 1}|V(y)|^{2} d y \leqq M \quad(n=1,2,3) \\
& \int_{|x-y| \leqq 1}|V(y)|^{2}|x-y|^{4-n-\alpha} d y \leqq M \quad(n \geqq 4)
\end{aligned}
$$

1) In the sequel, we shall use this notation in the case $X=L_{2}, L_{\infty}$ and $Q_{\alpha}$. 
for some positive constant $M$ dependent on $V$. Let $V \in Q_{\alpha}$. Then it is known (cf. $[6]$, Satz 4.2 ) that for any given $\varepsilon>0$, there exists a constant $C_{\varepsilon}$ such that

$$
\|V \phi\| \leqq \varepsilon\left\|H_{0} \phi\right\|+C_{\varepsilon} \mid i \phi \| \quad \text { for any } \phi \in C_{0}^{\infty},
$$

where the notation $\phi \in C_{0}^{\infty}$ means that $\phi$ is infinitely differentiable and has a compact support. By virtue of this inequality, the operator $-\Delta+V$ defined on $C_{0}^{\infty}$ is essentially selfadjoint, that is, its closure, which will be denoted by $H$, is selfadjoint. Moreover its domain $D(H)$ coincides with $D\left(H_{0}\right)$, (3) holds for $\phi \in D(H)$, and the graph norms of $H_{0}$ and $H$ are equivalent; cf. [2], Chap. V, Theorem 4.5.

We shall prove the following

Theorem 1. Let $V$ be a real function of class $\varepsilon_{L_{\infty}}^{1}$ and repulsive. In case $n=1$ and 2, we assume in addition that there exist constants $a, b$ and $m$ such that $0<a<b$,

$$
m>a^{-1}(b-a)^{-2},
$$

and

$$
\left\{\begin{array}{l}
\partial V / \partial r \leqq-m \quad \text { in } \quad a \leqq r \leqq b \\
\partial V / \partial r<0 \quad \text { in } \quad b \leqq r
\end{array} \quad(n=1)\right.
$$

$$
\left\{\begin{array}{l}
\partial V / \partial r+\frac{1}{2} r^{-3} \leqq-\frac{1}{2} m \quad \text { in } \quad a \leqq r \leqq b \\
\partial V / \partial r+\frac{1}{2} r^{-3}<0 \quad \text { in } \quad b \leqq r .
\end{array}(n=2)\right.
$$

Then $H$ is absolutely continuous.

Corollary 1. Let $V$ satisfy the assumptions of Theorem 1 and

$$
\lim _{|x| \rightarrow \infty} \int_{|x-y| \leqq 1}|V(y)|^{2} d y=0 \text {. }
$$

Then the spectrum of the absolutely continuous operator $H$ is the interval 
$[0, \infty)$

Corollary 2. Assume that $V=V_{1}+V_{2}$ satisfies the following conditions;

i) Each $V_{i}$ is of class $\varepsilon_{Q_{\alpha}}^{2(k-1)}$, where $k$ is an integer strictly larger than $n / 4$.

ii) For large $r$, say for $r \geqq R, V_{1}$ is of class $\varepsilon_{L_{\infty}}^{1}$ and $\partial V_{1} / \partial r \leqq 0$ $(n \neq 2), \partial V_{1} / \partial r+\frac{1}{2} r^{-3} \leqq 0 \quad(n=2)$.

iii) $V_{1}$ satisfies (7) with $V$ replaced by $V_{1}$.

iv) $V_{2} \in L_{1}$.

Then the absolutely continuous spectrum of $H=-\Delta+V$ is $[0, \infty)$.

\section{Preliminaries}

In this section we assume that $V \in \varepsilon_{L_{\infty}}^{1}$ so that $V \in Q_{\alpha}$.

Let $P_{j}$ 's $(j=1,2, \ldots, n)$ be the differential operators given by

$$
P_{j} \phi=-i \partial \phi / \partial x_{j}
$$

with domain $D\left(P_{j}\right)=\varepsilon_{L_{2}}^{1}$. Then $P_{j}$ maps its domain into $L_{2}$ and $H_{0}=\sum_{i=1}^{n} P_{j}^{2}$.

Let $[A, B]$ be the commutator $A B-B A$ in the strict operator theoretical sense. If $f \in \varepsilon_{L_{\infty}}^{1}$, then we have

$$
i\left[P_{j}, f\right] \phi=\left(\partial f / \partial x_{j}\right) \phi \quad \text { for } \phi \in \varepsilon_{L_{2}}^{1} .
$$

Let $g_{j}$ 's $(j=1,2, \ldots, n)$ be real valued functions of class $\varepsilon_{L_{\infty}}^{1}\left(R^{n}\right)$ and put

$$
A=(H-i)^{-1}\left(\sum_{j=1}^{n}\left(g_{j} P_{j}+P_{j} g_{j}\right)\right)(H+i)^{-1}
$$

Since $\left(g_{j} P_{j}+P_{j} g_{j}\right)(H+i)^{-1}$ is bounded by the closed graph theorem, $A$ and $H A$ are bounded so that $A H C(H A)^{*}$ is also bounded on $D(H)$. Thus the operator $i[H, A]$ is defined on $D(H)$ and bounded. Put $C=$ $i\left(H A-(H A)^{*}\right)$. Then it is bounded and selfadjoint and the closure of 
$i[H, A]$. Since $[H, A]=-i C$ on $D(H)$ and $A$ is bounded, the following lemma is a special case of a theorem of Putnam [4, Theorem 2.13.2].

Lemma 1. If there exists an operator $A$ such that the operator $C$ is nonnegative and 0 is not an eigenvalue of $C$, then $H$ is absolutely continuous.

In the next section we shall construct such $g_{j}$ 's that the operator $A$ defined by (9) satisfies the assumptions of Lemma 1 .

Lemma 2. Let $g(r)$ be a real function defined on the half line $r \geqq 0$ of class $\varepsilon_{L_{\infty}}^{3}(0, \infty)$ such that $g(r)=$ const $r$ for small $r$. Put

$$
g_{j}(x)=g(|x|) x_{j} /|x| \text {. }
$$

Then we have for $\phi \in L_{2}$,

$$
\begin{aligned}
& C \phi=4 \sum_{j, k=1}^{n}(H-i)^{-1} P_{j} x_{j} x_{k} g^{\prime} r^{-2} P_{k}(H+i)^{-1} \phi+ \\
& +4 \sum_{j, k=1}^{n}(H-i)^{-1} P_{j}\left(\delta_{j k}-x_{j} x_{k} r^{-2}\right) g r^{-1} P_{k}(H+i)^{-1} \phi- \\
& \quad-(H-i)^{-1} G(x)(H+i)^{-1} \phi
\end{aligned}
$$

where $r=|x|$,

$$
\begin{aligned}
G(x) & =g^{\prime \prime \prime}+2(n-1) r^{-1} g^{\prime \prime}+(n-1)(n-3) r^{-3}\left(r g^{\prime}-g\right)+2 g \partial V / \partial r \\
g & =g(|x|) \text { and }{ }^{\prime}=d / d r
\end{aligned}
$$

Proof. We note thet $g_{j} \in \varepsilon_{L_{\infty}}^{3}$. Let $\phi \in C_{0}^{\infty}$. Then $\left(g_{j} P_{j}+P_{j} g_{j}\right) \phi$ are of class $\varepsilon_{L_{\infty}}^{2}$ and have compact supports so that they belong to $D\left(H_{0}\right)$, and we have

$$
\begin{array}{r}
i\left[H_{0}, \sum_{j=1}^{n}\left(g_{j} P_{j}+P_{j} g_{j}\right)\right] \phi=\sum_{j, k=1}^{n} i\left[P_{k}^{2}, g_{j} P_{j}+P_{j} g_{j}\right] \phi \\
=\sum i\left\{P_{k}\left[P_{k}, g_{j} P_{j}+P_{j} g_{j}\right]+\left[P_{k}, g_{j} P_{j}+P_{j} g_{j}\right] P_{k}\right\} \phi \\
=\sum i\left\{P_{k}\left(\left[P_{k}, g_{j}\right] P_{j}+g_{j}\left[P_{k}, P_{j}\right]+\left[P_{k}, P_{j}\right] g_{j}+P_{j}\left[P_{k}, g_{j}\right]\right)+\right.
\end{array}
$$




$$
\begin{aligned}
& \left.+\left(\left[P_{k}, g_{j}\right] P_{j}+g_{j}\left[P_{k}, P_{j}\right]+\left[P_{k}, P_{j}\right] g_{j}+P_{j}\left[P_{k}, g_{j}\right]\right) P_{k}\right\} \phi \\
= & \sum i\left\{P_{k}\left[P_{k}, g_{j}\right] P_{j}+P_{k} P_{j}\left[P_{k}, g_{j}\right]+\left[P_{k}, g_{j}\right] P_{j} P_{k}+P_{j}\left[P_{k}, g_{j}\right] P_{k}\right\} \phi,
\end{aligned}
$$

where we used the identities $\left[P_{j}, P_{k}\right]=0$. Using (8) with $f$ replaced by $g_{j}$, we have

$$
\begin{aligned}
& i\left[H_{0}, \sum\left(g_{j} P_{j}+P_{j} g_{j}\right)\right] \phi \\
= & \sum\left\{P_{k} \partial g_{j} / \partial x_{k} P_{j}+P_{j} \partial g_{j} / \partial x_{k} P_{k}+P_{j} P_{k} \partial g_{j} / \partial x_{k}+\partial g_{j} / \partial x_{k} P_{k} P_{j}\right\} \phi \\
= & \sum\left\{2\left(P_{k} \partial g_{j} / \partial x_{k} P_{j}+P_{j} \partial g_{j} / \partial x_{k} P_{k}\right)+P_{j}\left[P_{k}, \partial g_{j} / \partial x_{k}\right]-\right. \\
& \left.-\left[P_{k}, \partial g_{j} / \partial x_{k}\right] P_{j}\right\} \phi \\
= & \sum\left\{2\left(P_{k} \partial g_{j} / \partial x_{k} P_{j}+P_{j} \partial g_{j} / \partial x_{k} P_{k}\right)+\left[P_{j},\left[P_{k}, \partial g_{j} / \partial x_{k}\right]\right]\right\} \phi \\
= & 2 \sum\left\{P_{k} \partial g_{j} / \partial x_{k} P_{j}+P_{j} \partial g_{j} / \partial x_{k} P_{k}\right\} \phi-\left\{\Delta\left(\sum_{j} \partial g_{j} / \partial x_{j}\right)\right\} \phi .
\end{aligned}
$$

On the other hand

$$
i\left[V, \sum\left(g_{j} P_{j}+P_{j} g_{j}\right)\right] \phi=-2\left(\sum g_{j} \partial V / \partial x_{j}\right) \phi
$$

Thus we have

$$
\begin{aligned}
i\left[H, \sum_{j}\left(g_{j} P_{j}+P_{j} g_{j}\right) \phi=\right. \\
=2 \sum_{j, k}\left\{P_{k} \partial g_{j} / \partial x_{k} P_{j}+P_{j} \partial g_{j} / \partial x_{k} P_{k}\right\} \phi- \\
\quad-\left\{\Delta\left(\sum_{j}\left(\partial g_{j} / \partial x_{j}\right)+2 \sum_{j} g_{j} \partial V / \partial x_{j}\right\} \phi\right.
\end{aligned}
$$

for $\phi \in C_{0}^{\infty}$.

Let $\phi$ and $\psi$ be such that $(H+i)^{-1} \phi,(H+i)^{-1} \psi \in C_{0}^{\infty}$. Then $\phi$ and $\psi$ run over a dense set since $H$ restricted on $C_{0}^{\infty}$ is essentially selfadjoint. Noting that $\sum\left(g_{j} P_{j}+P_{j} g_{j}\right)(H+i)^{-1} \phi \in D(H)$ and using formula (13), we have

$$
\begin{aligned}
& (C \phi, \phi)=i\left(\left((H A)-(H A)^{*}\right) \phi, \phi\right) \\
& \quad=i\left((H-i)^{-1} H\left(\sum\left(g_{j} P_{j}+P_{j} g_{j}\right)\right)(H+i)^{-1} \phi, \psi\right)
\end{aligned}
$$




$$
\begin{aligned}
& -i\left(\phi,(H-i)^{-1} H\left(\sum\left(g_{j} P_{j}+P_{j} g_{j}\right)\right)(H+i)^{-1} \psi\right) \\
= & i\left(\left[H, \quad \sum\left(g_{j} P_{j}+P_{j} g_{j}\right)\right](H+i)^{-1} \phi, \quad(H+i)^{-1} \psi\right) \\
= & \left((H-i)^{-1} 2 \sum\left(P_{k} \partial g_{j} / \partial x_{k} P_{j}+P_{j} \partial g_{j} / \partial x_{k} P_{k}\right)(H+i)^{-1} \phi, \psi\right) \\
& -\left((H-i)^{-1}\left\{\Delta\left(\sum \partial g_{j} / \partial x_{j}\right)+2 \sum g_{j} \partial V / \partial x_{j}\right\}(H+i)^{-1} \phi, \psi\right) .
\end{aligned}
$$

The operator $C$ is bounded as was noted above. Since $\partial g_{\jmath} / \partial x_{k} \in \varepsilon_{L_{\infty}}^{2}$ and $\Delta\left(\sum \partial g_{j} / \partial x_{j}\right)+2 \sum g_{j} \partial V / \partial x_{j} \in L_{\infty}$, the two operators in the last member of this formula:

$$
\begin{aligned}
& C_{1}=2(H-i)^{-1}\left\{\sum\left(P_{k} \partial g_{j} / \partial x+P_{j} \partial g_{j} / \partial x_{k} P_{k}\right)\right\}(H+i)^{-1} \\
& C_{2}=(H-i)^{-1}\left\{\Delta\left(\sum \partial g_{j} / \partial x_{j}\right)+2 \sum g_{j} \partial V / \partial x_{j}\right\}(H+i)^{-1}
\end{aligned}
$$

are also bounded. Thus since $\phi$ and $\psi$ run over a dense set, we have

$$
C \phi=C_{1} \phi-C_{2} \phi \quad \text { for } \quad \phi \in L_{2}
$$

Now since $g_{j}(x)=g(r) x_{j} / r$, we have

$$
\partial g_{j} / \partial x_{k}=x_{j} x_{k} g^{\prime} r^{-2}+\left(\delta_{j k}-x_{j} x_{k} r^{-2}\right) g r^{-1}
$$

and

$$
\begin{aligned}
\Delta\left(\sum \partial g_{j} / \partial x_{j}\right) & =\left(d^{2} / d r^{2}+(n-1) r^{-1} d / d r\right)\left(g^{\prime}+(n-1) r^{-1} g\right)= \\
& =g^{\prime \prime \prime}+2(n-1) r^{-1} g^{\prime \prime}+(n-1)(n-3)\left(r^{-2} g^{\prime}-r^{-3} g\right) .
\end{aligned}
$$

Thus we have

$$
\begin{aligned}
C_{1}= & 4 \sum(H-i)^{-1}\left\{P_{j} x_{j} x_{k} g^{\prime} r^{-2} P_{k}+P_{j}\left(\delta_{j k}-x_{j} x_{k} r^{-2}\right) g r^{-1} P_{k}\right\}(H+i)^{-1}, \\
C_{2}= & (H-i)^{-1}\left\{g^{\prime \prime \prime}+2(n-1) r^{-1} g^{\prime \prime}+(n-1)(n-3) r^{-3}\left(r g^{\prime}-g\right)+\right. \\
& +2 g \partial V / \partial r\}(H+i)^{-1} \\
= & (H-i)^{-1} G(x)(H+i)^{-1}
\end{aligned}
$$

which with (14) proves the lemma. 
Lemma 3. Let $g(r)$ satisfy the assumptions of Lemma 2. Assume also that

$$
\begin{aligned}
& g \geqq 0, g^{\prime} \geqq 0, G \leqq 0 \quad(r \neq 0), \\
& g^{\prime}>0 \quad(r \leqq b), \quad G<0 \quad(r \geqq a),
\end{aligned}
$$

for some constants $a$ and $b(0<a<b)$. Then the operator $C$ is nonnegative and zero is not an eigenvalue of $C$.

Proof. First we show that $C \geqq 0$. We note the formula (15). We have $C_{2} \leqq 0$ since $G \leqq 0$ and $C_{1} \geqq 0$ since $g, g^{\prime} \geqq 0$ and the matrices $\left(x_{j} x_{k}\right)$ and $\left(\delta_{j k}-x_{j} x_{k} r^{-2}\right)$ are nonnegative. Thus we have $C=C_{1}-C_{2} \geqq 0$.

Next we show that zero is not an eigenvalue of $C$. If $C \phi=0$, then since the second term of (11) is nonnegative as was shown above, we have

$$
\begin{gathered}
0=(C \phi, \phi) \geqq 4 \sum\left((H-i)^{-1} P_{j} x_{j} x_{k} g^{\prime} r^{-2} P_{k}(H+i)^{-1} \phi, \phi\right) \\
-\left((H-i)^{-1} G(H+i)^{-1} \phi, \phi\right) \\
=4 \int\left|\sum x_{j} P_{j}(H+i)^{-1} \phi\right|^{2} g^{\prime} r^{-2} d x+\int G\left|(H+i)^{-1} \phi\right|^{2} d x \geqq 0,
\end{gathered}
$$

so that by virtue of $(17), u(x) \equiv\left((H+i)^{-1} \phi\right)(x)$ satisfies

$$
\partial u / \partial r(x)=i\left(\sum x_{j} r^{-1} P_{j}(H+i)^{-1} \phi\right)(x)=0 \text { for almost all }|x| \leqq b,
$$

and

$$
u(x)=0 \quad \text { for almost all }|x| \geqq a .
$$

Thus $u(x)=0$ for almost all $x$ since $u(x)=-\int_{1}^{a|| x \mid} \frac{d u(t x)}{d t} d t=0$ for $0<|x| \leqq a$. Thus we have $\phi=0$, which shows that zero is not an eigenvalue of the operator $C$.

\section{Proof of Theorem 1}

Now let us construct the function $g$ satisfying the assumptions of 
Lemma 3. Then by virtue of Lemma 1 , the proof of Theorem 1 will be completed.

First we treat the case $n \geqq 3$. Let $a$ and $b$ be some constants such that $0<a<b$. Let $k$ be a number such that $2<k<2(n-1)$. Put

$$
\begin{gathered}
g^{\prime \prime}(r)= \begin{cases}0 & (0 \leqq r \leqq a) \\
-\frac{b^{-k}}{b-a}(r-a) & (a \leqq r \leqq b) \\
-r^{-k} & (b \leqq r),\end{cases} \\
g^{\prime}(r)=-\int_{r}^{\infty} g^{\prime \prime}(r) d r
\end{gathered}
$$

and

$$
g(r)=\int_{0}^{r} g^{\prime}(r) d r
$$

Then

$$
g^{\prime \prime \prime}(r)= \begin{cases}0 & (0 \leqq r \leqq a) \\ -\frac{b^{-k}}{b-a} & (a \leqq r \leqq b) \\ k r^{-k-1} & (b \leqq r)\end{cases}
$$

is bounded. $g^{\prime \prime}$ is bounded and nonpositive. $g^{\prime}(r)$ is bounded and positive since $g^{\prime \prime} \in L_{1}(0, \infty)$ and $g^{\prime \prime} \leqq 0 . \quad g(r)$ is positive since $g^{\prime}>0$, and bounded since

$$
g(r)=\int_{0}^{b} g^{\prime} d r-(k-1)^{-1}(k-2)^{-1}\left(r^{-k+2}-b^{-k+2}\right) \quad \text { in } b \leqq r,
$$

and $k>2$.

Thus $g \in \varepsilon_{L_{\infty}}^{3}$ and $g$ satisfies (16) and (17) except the assertions on $G$. Now let us show that $G$ satisfies the assertions (16) and (17). We note that the third term $(n-1)(n-3) r^{-3}\left(r g^{\prime}-g\right)$ of $G$ in (12) is nonpositive since $\left(r g^{\prime}-g\right)^{\prime}=r g^{\prime \prime} \leqq 0$ and $\left(r g^{\prime}-g\right)(0)=0$. In $0 \leqq r \leqq a$, the first three terms in (12) are zero so that $G=2 g \partial V / \partial r \leqq 0$ by the assumption (1). In $a \leqq r \leqq b$, the first term $g^{\prime \prime \prime}$ is negative and the other terms are nonpositive so that $G<0$. In $b \leqq r$, 


$$
\begin{aligned}
G & \leqq g^{\prime \prime \prime}+2(n-1) r^{-1} g^{\prime \prime}=k r^{-k-1}-2(n-1) r^{-k-1} \\
& =(k-2(n-1)) r^{-k-1}<0
\end{aligned}
$$

since $k<2(n-1)$. Thus we have shown that this function $g$ is desired one in case $n \geqq 3$.

Next we treat the case $n=1,2$. Let $a$ and $b$ be the numbers in the assumption of Theorem 1 and $c$ be such that $a<c<b$. Put

$$
g(r)= \begin{cases}\frac{1}{2}(c-a)(b-c) r & (0 \leqq r \leqq a) \\ \frac{1}{2}(c-a)(b-c) r-\frac{b-c}{6(b-a)}(r-a)^{3} & (a \leqq r \leqq c) \\ \frac{1}{6}(a+b+c)(c-a)(b-c)+\frac{c-a}{6(b-a)}(r-b)^{3} & (c \leqq r \leqq b) \\ \frac{1}{6}(a+b+c)(c-a)(b-c) & (b \leqq r) .\end{cases}
$$

Then $g \in \varepsilon_{L_{\infty}}^{3}(0, \infty)$ and $g, g^{\prime},-g^{\prime \prime} \geqq 0$ for $r \geqq 0$ and $g^{\prime}>0$ for $r<b$. Let us show that $G \leqq 0(r \geqq 0)$ and $G<0(r \geqq a)$ for $c$ sufficiently near to $a$.

First let $n=1$. Then $G=g^{\prime \prime \prime}+2 g \partial V / \partial r$ and

$$
g^{\prime \prime \prime}= \begin{cases}0 & (0 \leqq r \leqq a) \\ -(b-c) /(b-a)<0 & (a \leqq r \leqq c) \\ (c-a) /(b-a)>0 & (c \leqq r \leqq b) \\ 0 & (b \leqq r)\end{cases}
$$

so that $G \leqq 0$ in $0 \leqq r \leqq a$ and $G<0$ in $a \leqq r \leqq c$ and $b \leqq r$ by the second assertion (5). In $c \leqq r \leqq b$, using the estimate $g(r) \geqq g(a)=\frac{1}{2}(c-a)(b-c) a$ and the assumption (5), we have

$$
\begin{aligned}
G & =(c-a) /(b-a)+2 g \partial V / \partial r \leqq(c-a) /(b-a)-(c-a)(b-c) a m \\
& =-(c-a)(b-c) a\left\{m-a^{-1}(b-c)^{-1}(b-a)^{-1}\right\}
\end{aligned}
$$


which is negative for $c$ sufficiently near to $a$ by the assumption (4). Thus (16) and (17) are verified for $n=1$.

Next let $n=2$. Since

$$
G=g^{\prime \prime \prime}+2 r^{-1} g^{\prime \prime}-r^{-2} g^{\prime}+r^{-3} g+2 g \partial V / \partial r,
$$

using the assumptions of Theorem 1, we have

$$
G \mid \begin{aligned}
& =2 g \partial V / \partial r \leqq 0 \quad(0 \leqq r \leqq a), \\
& \leqq g\left(r^{-3}+2 \partial V / \partial r\right) \leqq-m g<0 \quad(a \leqq r \leqq c), \\
& \quad g^{\prime \prime \prime}+g\left(r^{-3}+2 \partial V / \partial r\right) \leqq-(c-a)(b-c) a\left\{m-a^{-1}(b-c)^{-1}(b-a)^{-1}\right\} \\
& \quad(c \leqq r \leqq b), \\
& =r^{-3} g+2 \partial V / \partial r<0 \quad(b \leqq r),
\end{aligned}
$$

for $c$ sufficiently near to $a$. Thus (16) and (17) are now verified for $n=2$.

Thus we have ccnstructed the furction $g$ which have the desired properties, which yields Theorem 1 .

\section{Proof of Corollaries}

Proof of Corollary 1. Since the potential $V$ belongs to $Q_{\alpha}$ and satisfies the assumption (7), the essential spectrum of $H=H_{0}+V$ is $[0, \infty)$ (cf. [5]). On the other hand, by virtue of Theorem $1, H$ is absolutely continuous. Thus the spectrum of $H$ is $[0, \infty)$.

For the proof of Corollary 2, we use the following theorem due to Birman [1]:

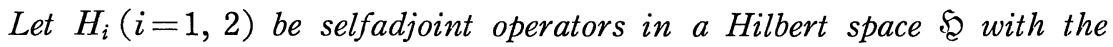
same domain. If the operator $\left(H_{2}+i\right)^{-k}\left(H_{2}-H_{1}\right)\left(H_{1}+i\right)^{-k}$ is of trace class for some positive number $k$, then the complete wave operators $W_{ \pm}\left(H_{2}, H_{1}\right)$ exist so that the absolutely continuous spectrum of $H_{1}$ and $H_{2}$ coincide with each other. (For the definition and natures of the wave operators, see e.g. [2], Chapter X.) 
Proof of Corollary 2. Let $V=V_{1}+V_{2}$ satisfy the assumptions of this corollary. First we note that we may assume without loss of generality that the function $V_{1}$ satisfies the assumptions of Corollary 1 with $V$ replaced by $V_{1}$. Indeed, let $h_{1}(r)$ and $h_{2}(r)$ be sufficiently smooth real functions of $r \geqq 0$ such that $h_{1}(r)=0(r \leqq R) ;=1(r \geqq 2 R)$, and $h_{2}(r)=1$ $\left(r \leqq \frac{1}{2} R\right), h_{2}^{\prime}(r)<0\left(\frac{1}{2} R<r \leqq 3 R\right)$ and $h_{2}(r)=e^{-r}(r \geqq 3 R)$, where $R$ is taken sufficiently large $\left(R>\frac{1}{3}\right)$ so that the assmption (ii) of this corollary is satisfied. Put

$$
\bar{V}_{1}=h_{1} V_{1}+c h_{2}, \quad \bar{V}_{2}=V_{2}+\left(1-h_{1}\right) V_{1}-c h_{2} .
$$

Then the assmptions of Corollary 2 with $V_{1}$ and $V_{2}$ replaced by $\bar{V}_{1}$ and $\bar{V}_{2}$, respectively, are satisfied and those of Theorem 1 with $V$ replaced by $\bar{V}_{1}$ are also satisfied for sufficiently large $c$.

Put $H_{1}=H_{0}+V_{1}$ and $H_{2}=H_{0}+V$. Then by virtue of the theorem of Birman stated above, it is sufficient to show that the operator $\left(\mathrm{H}_{2}+\right.$ $i)^{-k} V_{2}\left(H_{1}+i\right)^{-k}$ is of trace class for some $k$ since $H_{1}$ is absolutely continuous with spectrum $[0, \infty)$ by Corollary 1 .

We denote by $\hat{u}$ the Fourier transform of $u$. Since

$$
\left(\left(H_{0}+i\right)^{-k} u\right)(\xi)=\left(|\xi|^{2}+i\right)^{-k} \hat{u}(\xi),
$$

we have

$$
\left(\left(H_{0}+i\right)^{-k} u\right)(x)=(2 \pi)^{-n / 2} \int \exp (i \xi x) \hat{u}(\xi)\left(|\xi|^{2}+i\right)^{-k} d \xi
$$

Let $k$ be the integer in the assumption (i), that is, $k>n / 4$. Then $\exp (i \xi x)\left(|\xi|^{2}+i\right)^{-k} \in L_{2}$ so that we can apply the Parseval formula with the result that

$$
\left(\left(H_{0}+i\right)^{-k} u\right)(x)=\int K(x-y) u(y) d y
$$

where $K(x)=(2 \pi)^{-n / 2} \int \exp (i \xi x)\left(|\xi|^{2}+i\right)^{-k} d \xi \in L_{2}$. Thus the operator $V_{2}^{-\frac{1}{2}}\left(H_{0}+i\right)^{-k}$ is of the Hilbert-Schmidt class with the Hilbert-Schmidt norm $\left\|V_{2}\right\|_{L_{1}}\|K\|_{L_{2}}$ since $V_{2} \in L_{1}$ by the assumption (iv). 
As will be shown later (in Lemma 4), the operators $\left(H_{0}+i\right)^{k}\left(H_{1}+i\right)^{-k}$ and $\left(H_{0}+i\right)^{k}\left(H_{2}-i\right)^{-k}$ are bounded. Thus the operators

$$
V_{2}^{\frac{1}{2}}\left(H_{1}+i\right)^{-k}=V_{2}^{-\frac{1}{2}}\left(H_{0}+i\right)^{-k}\left(H_{0}+i\right)^{k}\left(H_{1}+i\right)^{-k}
$$

and

$$
\left(H_{2}+i\right)^{-k} V_{2}^{\frac{1}{2}} C\left(V_{2}^{\frac{1}{2}}\left(H_{2}-i\right)^{-k}\right)^{*}=\left(V_{2}^{\frac{1}{2}}\left(H_{0}+i\right)^{-k}\left(H_{0}+i\right)^{k}\left(H_{2}-i\right)^{-k}\right)^{*}
$$

are of the Hilbert-Schmidt class so that $\left(H_{2}+i\right)^{-k} V_{2}\left(H_{1}+i\right)^{-k}$ is of trace class. Thus we can complete the proof of Corollary 2 if we prove the following

Lemma 4. Let $V \in \varepsilon_{Q_{\alpha}}^{2(k-1)}$ and $H=H_{0}+V$. Then the operators $\left(H_{0}+i\right)^{k}(H \pm i)^{-k}$ are bounded.

Before proving this lemma, we prepare the following

Lemma 5. Let $V_{i} \in \varepsilon_{Q_{\alpha}}^{2(i-1)}$ and $\phi \in D\left(H_{0}^{k}\right)=\varepsilon_{L_{2}}^{2 k}$. Then for any $\varepsilon>0$ there exists a constant $C_{\varepsilon}$ such that the inequality

$$
\left\|\left(\prod_{i=1}^{k} V_{i}\right) \phi\right\| \leqq \varepsilon i \mid H_{0}^{k} \phi\left\|+C_{\varepsilon}\right\| \phi \|
$$

holds.

Proof. In case $k=1$, the inequality (18) is obvious by the inequality (3) and the assertion just after it. Let $\phi \in D\left(H_{0}^{k}\right)$. Since

$$
\begin{gathered}
(-\Delta)^{k-1} V_{k} \phi=\sum_{\substack{|\alpha|+|\beta ! \leqq 2(k-1)\\
| \beta \mid \neq 2(k-1)}} C_{\alpha, \beta}\left(D^{\alpha} V_{k}\right)\left(D^{\beta} \phi\right)+V_{k} H_{0}^{k-1} \phi, \\
\left\|\left(D^{\alpha} V_{k}\right)\left(D^{\beta} \phi\right)\right\| \leqq \mathrm{const}\left(\left\|H_{0} D^{\beta} \phi \mid !+\right\| \phi \|_{1}\right) \\
(|\alpha| \leqq 2(k-1),|\beta|<2(k-1))
\end{gathered}
$$

and

$$
\left\|V_{k} H_{0}^{k-1} \phi\right\| \leqq \varepsilon\left\|H_{0}^{k} \phi\right\|+C_{\varepsilon}\left\|H_{0}^{k-1} \phi\right\|,
$$

by virtue of (18) with $k=1$, we have that $V_{k} \phi \in D\left(H_{0}^{k-1}\right)$ 
and

$$
\left\|H_{0}^{k-1} V_{k} \phi\right\| \leqq \varepsilon\left\|H_{0}^{k} \phi\right\|_{1}+\sum_{|\beta| \leqq 2 k-1} C_{\varepsilon}\left\|D^{\beta} \phi\right\|^{\prime}
$$

Now we assume that the lemma holds with $k=1,2, \ldots, k-1$ by the assumption of induction. Since $V_{k} \phi \in D\left(H_{0}^{k-1}\right)$, we have

$$
\begin{aligned}
& \left\|\left(\prod_{i=1}^{k} V_{i}\right) \phi\right\|=\left\|\left(\prod_{i=1}^{k-1} V_{i}\right) V_{k} \phi\right\| \leqq \varepsilon\left\|H_{0}^{k-1} V_{k} \phi\right\|+C_{\varepsilon}\left\|V_{k} \phi\right\| \\
& \leqq \varepsilon\left\|H_{0}^{k-1} V_{k} \phi\right\|+C_{\varepsilon}\left(\left\|H_{0} \phi\right\|+\|\phi\|\right)
\end{aligned}
$$

by (18) with $k=k-1$ and $k=1$. The well known inequality

$$
\left\|D^{\beta} \phi\right\| \leqq \varepsilon\left\|H_{0}^{k} \phi\right\|+C_{\varepsilon}\|\phi\| \quad(|\beta| \leqq 2 k-1)
$$

and the inequalities (19) and (20) show that (18) holds with $k=k$, which yields the result by the induction method.

Proof of Lemma 4. Let $\phi \in C_{0}^{\infty}$. Then we have

$$
(-\Delta+V)^{k} \phi=\sum_{\substack{j \leqq k \\ \sum\left|\alpha_{i}\right|+|\beta|=2(k-j)}} C_{\alpha, \beta}\left(\prod_{i=1}^{j} D^{\alpha_{\imath}} V\right) D^{\beta} \phi=W \phi+H_{0}^{k} \phi,
$$

where

$$
W \phi=\sum_{\substack{0<j \leq k \\ \Sigma\left|\alpha_{\imath}\right|+|\beta| \\ \Sigma\left|\alpha_{i}\right| \neq 0}} C_{\alpha, \beta}\left(\prod_{i=1}^{j} D^{\alpha_{\imath}} V\right) D^{\beta} \phi+\sum_{j=1}^{k} V^{j} H_{0}^{k-j} \phi
$$

and $\alpha_{j}$ and $\beta$ are multi-indices. Since

$$
D^{\alpha_{2}} V \in \varepsilon_{Q_{\alpha}}^{2(k-1)-\left|\alpha_{i}\right|} C \varepsilon_{Q_{\alpha}}^{2(k-1)-\Sigma^{\left|\alpha_{i}\right|}}=\varepsilon_{Q_{\alpha}}^{2(j-1)+|\beta|} C \varepsilon_{Q_{\alpha}}^{2(j-1)},
$$

using (18) with $k=j$, we have

$$
\left\|\left(\prod_{i=1}^{j} D^{\alpha_{i}} V\right)\left(D^{\beta} \phi\right)\right\| \leqq \varepsilon\left\|H_{0}^{j} D^{\beta} \phi\right\|+C_{\alpha}\left\|D^{\beta} \phi\right\| \quad(2 j+|\beta| \leqq 2 k-1),
$$

and

$$
\left\|V^{j} H_{0}^{k-j} \phi\right\| \leqq \varepsilon\left\|H_{0}^{j} H_{0}^{k-j} \phi\right\|+C_{\varepsilon}\left\|H_{0}^{k-j} \phi\right\|^{-} \quad(1 \leqq j \leqq k) .
$$

By virtue of the inequality (21), we have 


$$
\|W \phi\| \leqq \varepsilon\left\|H_{0}^{k} \phi\right\|+C_{\varepsilon}\|\phi\| \quad \text { for } \phi \in C_{0}^{\infty} .
$$

Since $H^{k} \phi=H_{0}^{k} \phi+W \phi$ for $\phi \in C_{0}^{\infty}$ and (22) holds, it holds that $D\left(H^{k}\right)=$ $D\left(H_{0}^{k}\right)$ and

$$
\left\|H_{0}^{k} \phi\right\|+\|\phi\| \leqq \text { const }\left(\left\|H^{k} \phi\right\|+\|\phi\|\right), \quad \phi \in D\left(H_{0}^{k}\right),
$$

by virtue of the assertion just after the inequality (3).

The well known inequalities

$$
\left\|\left(H_{0}+i\right)^{k} \phi\right\| \leqq \operatorname{const}\left(\left\|H_{0}^{k} \phi\right\|+\|\phi\|\right)
$$

and

$$
\left\|H^{k} \phi\right\|+\|\phi\| \leqq \text { const }\left\|(H \pm i)^{k} \phi\right\|
$$

and the inequality (23) show that

$$
\left\|\left(H_{0}+i\right)^{k} \phi\right\| \leqq \text { const }\left\|(H \pm i)^{k} \phi\right\|
$$

which shows that the operators $\left(H_{0}+i\right)^{k}(H \pm i)^{-k}$ are bounded.

In conclusion, the author wishes to express his sincere gratitude to Professor T. Ikebe for his enduring encouragements and valuable discussions.

\section{References}

[1] Birman, M.S., A local criterion for the existence of wave operators, Dokl. Akad. Nauk. SSSR, 159 (1964), 485-488 (Russian); Soviet Math. Dokl. 5 (1964), 1505-1509 (English translation).

[2] Kato, T., Perturbation Theory for Linear Operators, Springer-Verlag, Berlin, 1966.

[3] Lavine, R.B., Absolute continuity of Hamiltonian operators with repulsive potentials, Proc. Amer. Math. Soc. 22 (1969), 55-60.

[4] Putnam, C.R., Commutation Properties of Hilbert Space Operators and Related Topics, Springer-Verlag, Berlin, 1967.

[5] Rejto, P., On the essential spectrum of the hydrogen energy and related operators, Pacific J. Math. 19 (1966), 109-140.

[6] Stummel, F., Singuläre elliptische Differentialoperatoren in Hilbertschen Räumen, Math. Ann. 132 (1956), 150-176. 
